\title{
Software Defined Networking and Network Function Virtualization for Service Providers using Network Slice AS a Service
}

\author{
K. Tamil Selvi, R.Thamilselvan
}

\begin{abstract}
The traditional network is configured based on the prescribed network requirements. Sometimes the resources of the network are underutilized and at sometimes there may resource starvation because of the static configuration of the network. As against traditional network, which is operated either as dedicated network or as an overlay network, network services can be operated over a shared network infrastructure. Thus maximum resource utilization under minimal infrastructure cost can be achieved. The on-demand network requirement can be configured dynamically using network slice. The backbone of the rapidly evolving 5G technology is network slice and service networks can be benefited from it. Different network function for multiple tenants can be enabled customized using network slice with each slice operating independently. Network slice can be offered as a service to meet various requirements from the network slice tenant with different granularities. The Software Defined Networking and Network Function Virtualization are the enabling technologies for network slice. This paper discusses various network slicing use case requirements. And also OpenFlow based software defined network environment is simulated to validate the discussions. Experimental results show that the efficiency of the service network is maximized with improved reliability of service.
\end{abstract}

Keywords: SDN, NFV, Network slice, 5G

\section{INTRODUCTION}

Real time services like real time interactive multimedia, high bandwidth video feed, etc., are sensitive to response times and amount of bandwidth. Failure to meet these criteria leads to service degradation. Technological advancements arise with Internet of Things, robotics, and interaction among them with new type of network. These verticals require more resource constraints and network connectivity. To manage the resource efficiently, the common network infrastructure has to be utilized among the available verticals. The service providers offer network capabilities in a standalone manner in a shared network environment upon the common network infrastructure. The requirements of individual tenants can be met using network slice. In order to create network slice, end-to-end partition of network infrastructure has to be done. The partitioned network infrastructure can be user equipment, access or core transport networks, edge and central data center resources. The instances of network slicing share a common network infrastructure, which provide flexible design of specific network functions.

Revised Manuscript Received on December 30, 2019.

* Correspondence Author

K. Tamil Selvi, Kongu Engineering College, Erode

Dr.R.Thamilselvan, Kongu Engineering College, Erode

(C) The Authors. Published by Blue Eyes Intelligence Engineering and Sciences Publication (BEIESP). This is an open access article under the CC BY-NC-ND license (http://creativecommons.org/licenses/by-nc-nd/4.0/)
The network function can be customized to support differentiated performance requirements of vertical industries. Customization of network function based on tenant requirements can be enabled through network slicing. The on-demand network requirements can be suited by creation of network slice customization. Decomposition of resources from an underlying network infrastructure and logically aggregates them as part of a slice is enabled by network slicing. The data speed, quality, reliability, latency, security, etc are customisable network capabilities.

Network slicing offers full flexibility and support for diverse performance requirements. This is achieved by the modularization and flexible composition of network functions. Also, the notion of network programmability is adopted. Network functions virtualization (NFV) and software defined networking (SDN) are used as enablers. The former allows, through the virtualization of Network Functions (NF), the achievement of a modular logical architecture and flexible placement of NFs throughout the network. The latter allows simplification of forwarding functions but, more importantly, a more advanced separation of control and user plane functionality. With these two enablers, the network can become fully programmable. SDN is a framework to solve a set of problems implying many solutions. An open API that provides a standard interface for programming the data plane switch is OpenFlow. Traditional networks are often quite a static, slow to change and dedicated to single services. With software-defined networking, different network services can be handled by the network dynamically. Thus multiple services are aggregated in one common infrastructure. This aggregation can be performed by centralized network control. The logical separation of data and control logic provides automation and coordination of multiple network services. The main driving factor for using SDN are efficiency, optimization of existing applications, services and infrastructure, scalability and innovation. The global network view provided by SDN controller remove the need to know where network resources are physically located, resource cost, organization and so on. Thousands of devices can be managed and controlled by orchestrator command. The network behaviour can be changed using programming interfaces. Automatic configuration of dynamically changing network condition can be automated by controller and APIs. Resource and network connectivity monitoring, traffic engineering, load balancing and multi-tenancy can be achieved using SDN. Network Function Virtualization encompasses different network functions within a virtual server. The network functions are deployed in a single virtual server rather than deploying different hardware for different network function. 


\section{Software Defined Networking and Network Function Virtualization for Service Providers using Network Slice AS A Service}

And also it separates functions like a firewall or encryption from dedicated hardware and moves the function to virtual servers. NFV also enhances programmability of the network.

The rest of this article is organized as follows. Section II explains the related works. The main characteristics of network slice are discussed in Section III. The industry requirements are discussed in Section IV. An architecture that realize network slice is shown in Section V. Network Slice Management Function and its functions are given in Section VI. The experimental setup and its evaluation are discussed in Section VII and VIII. This paper is concluded in Section IX.

\section{RELATED WORKS}

Designing a service specific slice is a complicated process. A fine grained and light weight service called microservice was proposed. A service can be combination of loosely coupled microservices which can be provided by service provider [1]. The service requirement can be divided into number of microservices and each microservice is delivered as network slice.

The performance of network slice can be monitored and adapted using management and organization building blocks [2]. Network slice subnet consists of Core Network part and Radio Access Network part. ONAP [3] stands for Open Network Automation Platform. The services can be automated, designed, orchestrated and managed by end users using virtual functions. It is the combination of the ECOMP (Enhanced Control, Orchestration, and Management \& Policy) and Open-O (Open Orchestrator). It brings the capabilities for designing, creating, orchestrating and handling of the full lifecycle management of Virtual Network Functions, Software Defined Networks, and the services.

Network slices are generated according to different services and user requirements and resources are reserved [8]. The network resource control scheme maximizes the resource utilization rate and diversifies network application. Slicing of network in the radio access network domain requires elasticity, efficient resource sharing and customization [9] because of scarce and limited frequency spectrum resources. Core network entities can be deployed on virtual platforms and virtual instances provides flexibility, elasticity and QoS assurance to the service provision.

\section{CHARACTERISTICS OF NETWORK SLICING}

The multiple logical networks can be created on the top of shared physical network infrastructure using virtualization. The created end-to-end network slice can also virtualizes not only network but also storage and compute also. The objective is to allow a physical mobile network operator to partition its network resources to allow for very different users, so-called tenants, to multiplex over a single physical infrastructure. The common physical infrastructure can be shared with for instance Internet of Things, Mobile Broadband and vehicular communications applications and these applications have different transmission characteristics. For example, Internet of Things encompasses a very large number of devices, but very low throughput. Mobile broad band will have a much smaller number of devices, but each one will be transmitting or receiving very high bandwidth content. At macro level, resources are configured based on need, each slice is isolated from each other and optimal traffic engineering among the slice can be performed for improved efficiency.

In upcoming 5G environment, new business opportunities are open for service provides with wide range of usability. The multiple logical slices created from the physical network infrastructure can be leased to multiple tenants by the service provider. A long term lease of network slice is needed for electrical utility that needs connectivity of its smart grid consist of sensors, meters, and controllers and optimize that slice for Internet of Things devices. A short term lease of network slice can be used for week long technical festival with requirement of streaming of high definition videos and voice over IP connectivity.

New business models like existing cloud and data center providers, such as Amazon and Google may use network slicing a new way to play in the mobile network space. High quality networking experience can be delivered by the existing mobile operators to lease network slices and concentrate mainly on their core strengths. But current physical infrastructure cannot be configured granularity of different tenants. Today's existing tenants have purely business and billing relationships with the network owner and run their tenants service over network overlays. Network slicing will allow the network owner to customize the compute, storage and networking functions of the infrastructure for a given virtual network operator's traffic characteristics.

There is a key difference between network slicing and Quality of Service (QoS). End-to end virtual network is given for a tenant by network slice whereas QoS is for specific type of network traffic. The existing QoS mechanisms like DiffServ can discriminate one type of traffic from other type of traffic, but cannot treat the same type of traffic from different tenants. Traffic isolation cannot be performed by DiffServ. And DiffServ for example cannot be applied for monitoring IoT traffic from a health monitoring network which have strict privacy and security requirements including where the data can be stored and who can access it. Since compute and storage aspect of network cannot be handled by DiffServ, the above shortfalls can be handled by network slice.

\section{VERTICAL REQUIREMENT USECASES}

\section{A. Augmented Reality / Virtual Reality}

Augmented Reality (AR) is a technique where the real world view is augmented, or assisted, by a computer generated views, this can be in single or multi-sensory modes. Virtual Reality (VR) is the technology to construct a virtual environment, which may be based on the real environment within which people could have real-time interaction.

Strong interactive VR [4] applications like audio-visual interactions, Low-delay speech and video coding can be provisioned using network slice. The network slice should consider different aspect of video codec like frame rate, resolution and bandwidth for good user experience. AR applications like assistance with complex tasks, connecting remote workers, accelerated learning, etc, can be impacted with improved efficiency through network slicing dynamically.

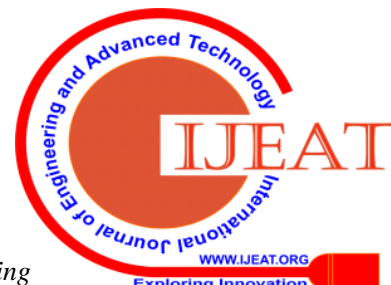




\section{B. Automotive}

Cellular Vehicle[4] to everything is considered as one of the most prominent use case of network slice. It needs ultra reliable and low latency communication and tailored network services based on customer requirement. The other services like infotainment, telematics, road safety and efficiency, advanced driving services, etc, requires predictive quality of services. Network slice through virtualization of network function helps to accommodate multiple tenants.

\section{Energy}

The energy industry has some very specific requirements on the supporting communication solutions that go beyond what current LTE can provide. The critical machine type communication (MTC) applications of energy grid protection and control and also to the massive volume of MTC type applications of the emerging smart metering can be supported by network services. Network slice could provide efficient and economical wireless solutions for applications like smart grid, micro-grids, smart meters and aggregate gateways, electricity traffic scheduling, etc,

\section{Healthcare}

The availability and usage of electronic processes[4] and communication technology has made health and wellness industry is about to change dramatically. Availability is the most important requirement of this vertical, followed by latency and then throughput. Remote surgery, remote healthcare, health and wellness monitoring, hospital, etc, needs different network requirement which can be provisioned using network slicing.

\section{E. Industry 4.0}

The "Fourth Industrial Revolution" or Industry 4.0[4] is set to fundamentally change the manufacturing industry. Flexibility, versatility, resource efficiency, cost efficiency, worker support, and quality of industrial production and logistics are the main drivers. The demanding requirement of use cases of Industry 4.0 are latency, synchronicity and availability.

Mobile business stake holders[4] define three categories of services and their characteristics such as eMBB (enhanced Mobile Broadband), mMTC (massive Machine Type Communications) and URLLC (UltraReliable and Low Latency Communications). To support these services, the key performance index such as user experience data rate, peak data rate, mobility, latency, connection density, energy efficiency, spectrum efficiency, and traffic volume density are elicited.

\section{NETWORK SLICE ARCHITECTURE}

The Network Slice (NS)-Subnet[5] denotes any physical or logical network resources. The business logic of the end user is represented by service. It may demand any resources or attributes in the form of network slice. The user can select an available slice or create a new slice based on the requirement. Network Slice repository stores slices with its properties and access rules. The user service has access to the repository to check for the presence of the required network slice. If the required slice doesn't exist, closest match slice

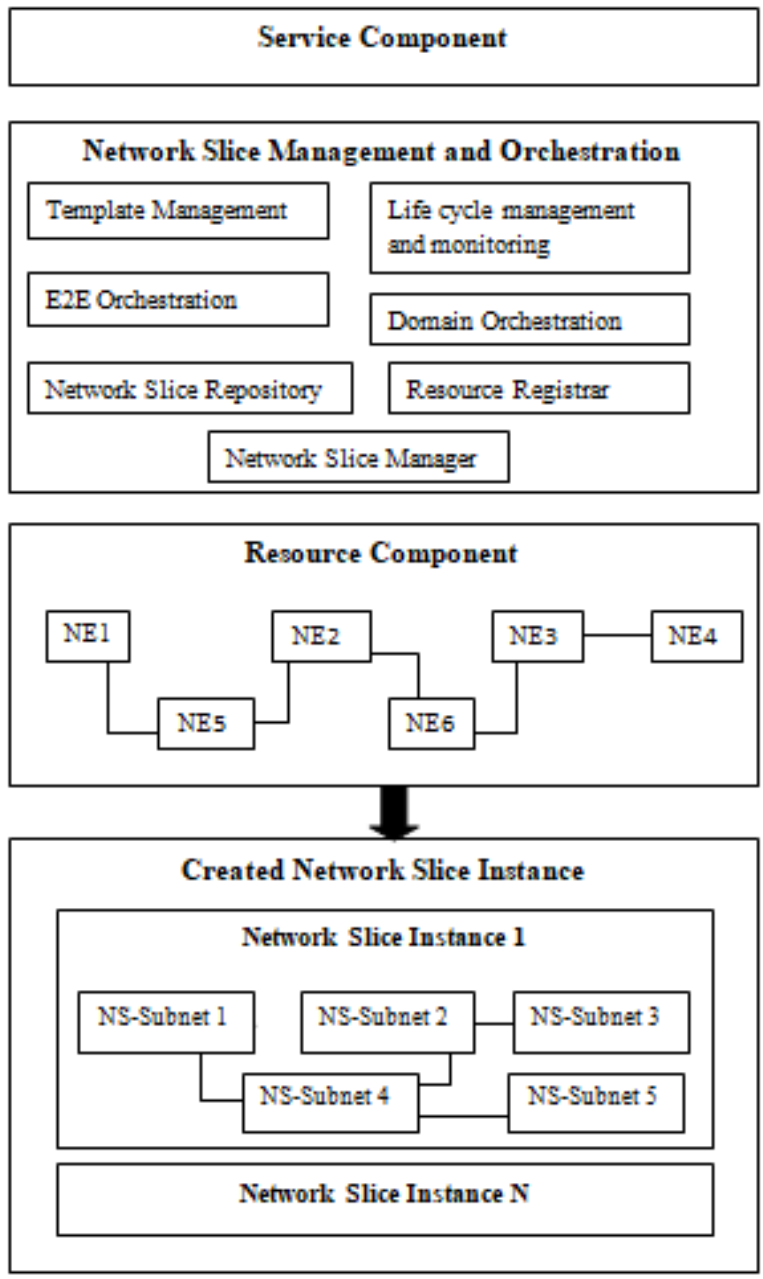

Fig. 1: Network Slice Architecture

will be attached to the service. New slice may be created and deployed by the service. The service is attached to the slice and the data forwarding mechanism for end user traffic will be configured automatically by the configuration rules.

Network slice[7] has life cycle to be managed since it is a dynamic entity. The creation, updation and deletion of network slice is managed by orchestrator. Orchestration is a system functions that automate the network functions in slices autonomically coordinate the slices lifecycle. The components of network slice shown in Figure 1 are coordinated to ensure an optimized allocation of the necessary resources across the network. It is a continuous optimization process of allocating resources to satisfy contending demands. The optimization would include service level agreement commitments. Many factors such as customer endpoint location, geographic or topological proximity, delay, aggregate or fine-grained load also contribute to optimization.

The mapping of requirements from orchestrator to network resources[12] and managing these resources of a specific network slice instance is done by network slice manager. In heterogeneous environment, end-to-end deployment of network services can be done in fast, dynamic and automated manner. All access permission and interaction between network slice and external function are managed by network slice manager.

Published By: 


\section{Software Defined Networking and Network Function Virtualization for Service Providers using Network Slice AS A Service}

\section{NETWORK SLICE MANAGEMENT FUNCTION}

The different objectives of Network Slice Management Function (NSMF) are

1. Maximum utilization of the network components

2. Decreased the average delay time from slice request to slice activation

3. Decreased the number of rejected slice requests

4. Maximize the number of slices with high throughput.

A network slice[8] instance can be represented as $\mathrm{NSI}=\left\{c, a, s, t_{s}, t_{w}\right\}$, where $c$ is a core network component, $a$ is access network component, $s$ is network slice, $t_{s}$ is remaining life time of network slice and $t_{w}$ is waiting time before slice was activated. The initial request for network slice is $=\left\{c, a, s, t_{s}, 1\right\}$.

At time $t$, the number of network slice[11] resources scheduled by NSMF is given by function, $X(t), t=1, \ldots$, For the time period $\left[T_{1}, T_{2}\right]$, an average utilization of the network components for the NSMF model given by the $A_{[\mathrm{T} 1, \mathrm{~T} 2]}$. Let $\delta(\mathrm{t})$ be the function that denotes average delay units that network slice requests issued at time $t$ and it waits till activation of the slice.

$A_{\left[T_{1}, T_{2}\right]}=\frac{1}{T_{2}-T_{1}} \sum_{t=T_{1}}^{T_{2}} X(t)$

The utilization ratio function $\mathrm{U}(\mathrm{t})$, is given by

$$
U(t)=\frac{X(t)}{\delta(t)}
$$

For the time period $\left[\mathrm{T}_{1}, \mathrm{~T}_{2}\right]$, average utilization ratio $\mathrm{UR}_{[\mathrm{T} 1, \mathrm{~T} 2]}$ is given by

$U R_{\left[T_{1}, T_{2}\right]}=\frac{1}{T_{1}-T_{2}} \sum_{t=T_{1}}^{T_{2}} U(t)$

The objective function can be written as

\section{Maximize $U(t)$ in the interval $\left[T_{1}, T_{2}\right]$}

\section{EXPERIMENTAL SETUP}

The OpenStack is used as Virtual Infrastructure Manager used for managing and controlling network function virtual instances. Open Baton is used as Network Function Virtualization Orchestrator. The Slice orchestrator is implemented using Django web development framework. It runs in Docker container on physical machine that is configured to be the compute node. It is a web server capable of communicating with the Open Baton Network Function Virtualization Orchestrator, the slice selector, the DNS server and Open vSwitch. The slice orchestrator provides REST API for triggering new slice creation and virtual network functions. Figure 2 shows the test environment with different slices namely common slice, ondemand video slice, IoT slice and Streaming video slice. When there is any change in the network condition or an event is triggered in the network, a new slice will be created.
The request for new slice creation will be sent to slice orchestrator. Then it sends request to OpenBaton which creates network service identifier. With this identifier, Slice orchestrator sends request to OpenStack to instantiate virtual network function based on the requirement.

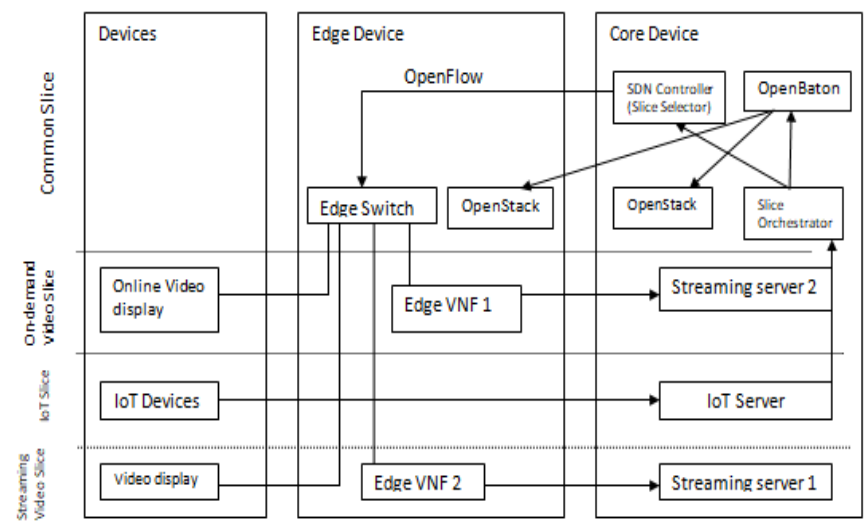

Fig. 2: Experimental Topology

The virtual network function can be pre-installed image or normal image. The QoS agent will provide the needed network resource requirement. Then the service will be active after tunnelling with the edge devices.

\section{PERFORMANCE EVALUATION \& RESULT}

The evaluation criteria used to test the performance of the network in this case is how much time it takes to set up slice after an event is triggered. The setup time is evaluated based on two different images of each virtual network function. One is normal image which is host operating system image which does not contain any pre-installed virtual network function related software. The other is pre-built image that has all virtual network function software.

The slice setup time using normal image is greater than that of the pre-built image using SDN. Thus for critical application, the efficiency of the network can be improved. When the network request cannot be served within the threshold time, the reliability of the network also reduced. The pre-built image provides faster network services based on the dynamic need of the requirement. From the Figure 3 it is clear that response time of the network for any event is reduced by a factor of 3 . As the number of parameters for the slice increases, the optimization using network function virtualization provides better network utilization.

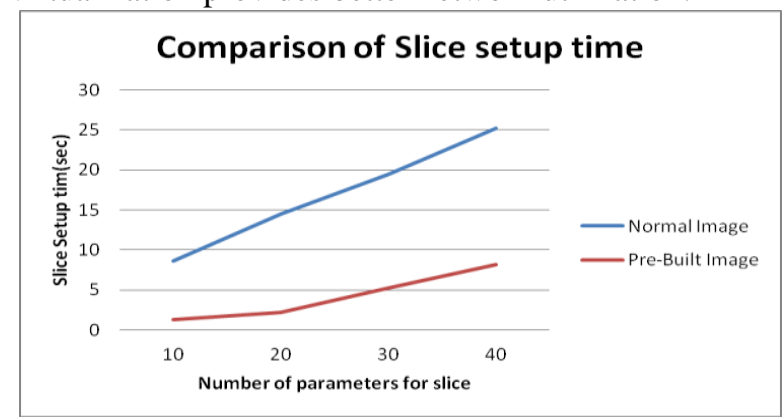

Fig 3: Comparison of slice setup time

Published By:

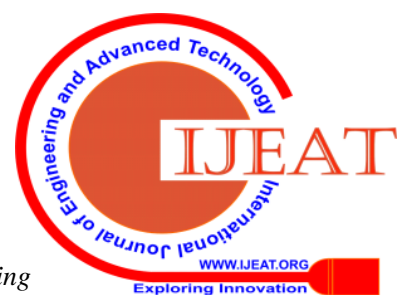

\& Sciences Publication 


\section{CONCLUSION}

The diverse and increased demand for network services cannot be met with the existing technologies. And also it is important provide network slice that are optimized for individual services rapidly. With software define networking and network function virtualization, the enablers of 5G networks, it is possible to provide reliable service for critical network applications. The efficiency of the network is also improved by the optimized service provision. The response time of the network provides indications on congestion, throughput, delay and other network parameters. This work provides the framework for reliable data services in high speed heterogeneous network. Further this framework can be extended with multiple controllers for network resilience.

\section{REFERENCES}

1. Minami, Yuki, et al. "An architecture and implementation of automatic network slicing for microservices." NOMS 2018-2018 IEEE/IFIP Network Operations and Management Symposium. IEEE, 2018.

2. Kaloxylos, Alexandros. "A survey and an analysis of network slicing in 5G networks." IEEE Communications Standards Magazine 2.1 (2018): 60-65.

3. Lee, Wangbong, Taeheum Na, and Jeongyun Kim. "How to Create a Network Slice?-A 5G Core Network Perspective." In 2019 21st International Conference on Advanced Communication Technology (ICACT), pp. 616-619. IEEE, 2019.

4. Network Slicing Use Case Requirements April 2018, 2018 GSM Association

5. Wang, Shuqi, Bei Liu, and Yu Feng. "Design of Multi-service Network Slicing Scheme Based on SDN/NFV." In 2018 International Conference on Sensor Networks and Signal Processing (SNSP), pp. 344-351. IEEE, 2018.

6. Afolabi, Ibrahim, Tarik Taleb, Konstantinos Samdanis, Adlen Ksentini, and Hannu Flinck. "Network slicing and softwarization: A survey on principles, enabling technologies, and solutions." IEEE Communications Surveys \& Tutorials 20, no. 3 (2018): 2429-2453.

7. Alliance, N. G. M. N. "Description of network slicing concept." NGMN 5G P 1 (2016): 1.

8. https://tools.ietf.org/id/draft-netslices-usecases-01.html

9. https://tools.ietf.org/id/draft-geng-netslices-architecture-01.html

10. Zhang, Haijun, Na Liu, Xiaoli Chu, Keping Long, Abdol-Hamid Aghvami, and Victor CM Leung. "Network slicing based 5G and future mobile networks: mobility, resource management, and challenges." IEEE Communications Magazine 55, no. 8 (2017): 138 145.

11. Americas, G. "Network slicing for 5 g network and services." In Tech. Rep. 2016. 